\title{
LOS SUELOS DE AREAS INUNDABLES DE LA \\ AMAZONIA PERUANA: POTENCIAL, \\ LIMITACIONES Y ESTRATEGIA PARA SU \\ INVESTIGACION
}

RODRIGUEZ ACHUNG FERNANDO ( $\left.{ }^{*}\right)$

\section{INTRODUCCION}

La amazonía peruana, conocida también con el nombre de selva, montaña, región oriental, región amazónica o hílea amazónica, abarca una extensión de $759,052 \mathrm{~K} \mathrm{~m}^{2}$ que, representa aproximadamente el $59 \%$ del territorio nacional y cerca del $14 \%$ de la amazonia continental. Su población, estimada a 1990, es aproximadamente de 2`450,000 habitantes.

Desde el punto de vista climático, la amazonia peruana, en términos generales, se caracteriza por presentar el evadas temperaturas y fuertes precipitaciones, la temperatura media anual varía de 22.5 a 27.2 ㅇ C y la precipitación media anual de 602 a $3,411 \mathrm{~mm}$.

En. la amazonía peruana se ha identificado tres grandes ecosistemas: el bosque pluvial, que se caracteriza por una estación seca, de no más de 3 meses consecutivos y presentar una vegetación natural típica de bosque húmedo tropical; el bosque estacional semisiempreverde, constituido por una pronunciada época seca, no mayor de 4 meses consecutivos; presenta una vegetación natural típica de bosque seco tropical; y el bosque espinoso, que se caracteriza por su baja precipitación y por ser una región árida (Sánchez y Benites, 1983).

En esta región, se distinguen principalmente dos zonas diferenciadas por su altitud, morfología, clima, características de sus ríos, etc., que son la selva alta y selva baja. 
La selva alta es la zona del piedemonte oriental de la Cordillera de los Andes, se encuentra entre 2,500 y 500 m.s.n.m., es escarpada y presenta cordilleras, cerros y colinas, así como valles anchos y de gran longitud, siendo su geología compleja. Representa aproximadamente el $27 \%$ del Perú.

La selva baja se sitúa por debajo de los 500 m.s.n.m. y se inicia al finalizar los últimos contrafuertes andinos. M orfológicamente, se distinguen dos unidades fisiográficas: la primera, incluye terrazas bajas, sujetas a inundaciones, de origen aluvial reciente y de mayor fertilidad; según ONERN (1982), estas tierras comprenden una superficie aproximada de 3'278,50\{) Ha. La segunda, está constituida por terrazas no inundables y superficies onduladas, con diferentes grado de disección, en sedimentos no consolidados del pleistoceno y del terciario (Zamora 1987; Dumont y García, 1989).

En las terrazas bajas inundables de los ríos A mazonas, U cayali y M arañón, se sitúa una parte significativa de la población rural y la mayor parte de la superficie dedicada a la producción de cultivos alimenticios. No obstante esta situación, ha sido muy escaso el esfuerzo de las instituciones de investigación en ampliar el conocimiento sobre estas áreas y generar tecnologías adecuadas para su uso racional.

El presente trabajo, trata de sistematizar y analizar la información disponible, con el objeto de plantear una estrategia general para la investigación y desarrollo de los suelos local izados en áreas inundables de la selva baja del Perú.

\section{El agua y ecosistemas típicos}

Cerca de 7'000,000 de $\mathrm{Km}^{2}$ de tierras son drenadas por el río Amazonas, produciéndose una descaiga promedio anual de 175,000 M 3 S1 de agua en el Océano A tlántico, que representa 1/5 $61 / 6$ de todas las aguas de los ríos de los continentes (Sioli, 1984). De allí es que el agua juega un rol preponderante en el paisaje amazónico (Junk, 1983).

Desde este punto de vista, y tal como lo propone M eggers (1976), en su estudio sobre la amazonía, es posible reconocer dos subregiones de tamaño marcadamente contrastante y que difieren en su potencial de subsistencia: la basta "tierra firme', en donde los recursos están muy dispersos pero continuamente disponibles y la estrecha llanura de inundación, denominada "várzea" en el Brasil, en donde alternan la escasez y la abundancia, según suba o baje el nivel del río. 
Desde el punto de vista ecológico, la "tierra firme", localizada en terrazas altas, colinas y montañas, constituye un ecosistema cerrado, es decir, que el ciclo de nutrientes se da fundamentalmente entre el suel o y la biomasa que soporta.

En cambio, la "várzea", localizada en áreas inundables, constituye un ecosistema abierto. Según Junk (1983), la várzea es comparable a un gran transformador biológico, pues, estas áreas son anualmente fertilizadas por la inundación y, después de haber existido un equilibrio ecológico, devuelven al río una cantidad de nutrientes equivalentes a aquellos recibidos en forma inorgánica, como sales minerales disueltas y sedimentos. En la várzea, ellos son transformados parcialmente por medio de la energía solar en materia orgánica y devueltos al río en forma de plantas acuáticas, detritos orgánicos, troncos de árboles, sustancias orgánicas disueltas, etc.

\section{L a cuenca del A mazonas y características de sus ríos}

El río A mazonas es el más grande de nuestro planeta, tanto por el área de su cuenca, como por su volumen. Su cuenca, en el Perú, abarca todo el territorio localizado al este de la Cordillera de los A ndes, exceptuando la cuenca del Titicaca. EI A mazonas, que en territorio peruano posee una longitud aproximada de 3,335 km., llega a tener casi $6,500 \mathrm{~km}$. en su desembocadura en el A tlántico.

En su trayecto recibe el aporte de numerosos tributarios, que poseen dos regímenes hidrológicos bien definidos: uno, en la parte norte, que incluye los ríos que nacen en las vertientes de los Andes ecuatoriales (Putumayo, Napo, Tigre, Pastaza, etc.) y otro, en la parte sur, que incluye a los ríos que nacen en las vertientes de los A ndes peruanos ( $M$ arañón, U cayali, etc.). Estos presentan sus fases hidrológicas casi opuestas, pues cuando los primeros están en época de vaciante, los segundos están en época de creciente. El ciclo hidrológico anual de los ríos que nacen en los A ndes del Perú y que son los de mayor caudal presentan cuatro fases bien marcadas: creciente (marzo, abril y mayo), media creciente (junio y julio), vaciante (agosto, setiembre y octubre) y media vaciante (noviembre, diciembre, enero y febrero). (M ontreuil et al, 1984 y A zabache et al, 1983).

Las diferencias de nivel entre creciente y vaciante en el río A mazonas son bastante marcadas. Así, en la ciudad de Manaos (Brasil), se ha registrado una variación media de $10 \mathrm{~m}$. por año (valor medio de 80 años) y la diferencia entre los niveles máximos y mínimos son de 8 a 15 m. (Junk, 1985) En Iquitos, M aco (1990), reporta una variación media de $8 \mathrm{~m}$. para el período 1962-1989, variando entre 5.33 y 10.48 m. Sin embargo, Angeles et al (1969), en el período 1933-1962, encontró una amplitud máxima absoluta de $11.4 \mathrm{~m}$. para esta misma ciudad. 
No todos los ríos de la cuenca amazónica poseen las mismas características limnológicas. Estudios realizados por el Instituto Max Planck, en convenio con el Instituto Nacional de Investigaciones de la Amazonía del Brasil, citado por Junk (1983), determinan la existencia de ríos de tres tipos de agua: de 'agua blanca', que tienen su origen en la región Andina o pre-A ndina, como el propio A mazonas, Purús, $M$ adeira, etc., que poseen una carga muy alta de sedimentos, ricos en sales minerales, con una composición química casi neutra (pH 6.5 a 7.0) y cantidades relativamente grandes de calcio y magnesio. De "agua negra", que nacen en los escudos de Guyanas y del Brasil central, como el río Negro; poseen poco material en suspensión, su color procede de la descomposición del material orgánico (ácidos húmicos y fúlvicos). Estas aguas son ácidas, con un pH alrededor de 4.0. De "agua clara", que son transparentes y transportan poco material en suspensión. Los valores de pH pueden variar entre 4.5 a más de 7.0. Para el Perú, el Instituto de Investigaciones de la A mazonía Peruana, reporta tendencias limnológicas similares para estos diferentes ambientes lóticos, tal como se observa en el Cuadro $\mathrm{N} \cong 1$ (M ontreuil et al, 1990).

Desde el punto de vista de las actividades económicas y de la localización de los asentamientos poblacionales, los sectores de ríos de "agua blanca" son los más importantes. Estos ríos transportan sedimentos ricos en sales minerales. Según M eade (1979), las cifras estimadas, publicadas en las décadas del 60 y el 70, indican que la cantidad de sedimentos que el río A mazonas lanza al mar varía entre 0.4 a $1.0 \times 10^{6}$ toneladas por año. El mismo autor, reporta para 1977, cifras de $1.7 \times 10^{6}$ toneladas por año en la ciudad de Iquitos, mientras que en Obidos (Brasil), una cifra superior de $4.7 \times 10^{6}$ toneladas por año.

El cauce o lecho menor del río A mazonas en el territorio peruano varía entre 2 y $5 \mathrm{~km}$, mientras que el lecho mayor de inundación es muy amplio, abarcando hasta $20 \mathrm{~km}$. de ancho. En el Brasil, M eggers (1976), reporta un ancho que varía entre 25 y $50 \mathrm{Km}$. hasta alcanzar en el "delta" un máximo de $200 \mathrm{~km}$.

Estas características se deben a que los terrenos localizados por encima de sus cauces, son casi planos o pocos accidentados y las aguas que los sobrepasan durante las crecientes se extienden rápidamente y recubren grandes sectores de estas Ilanuras amazónicas (Peñaherrera, 1985).

La mayor parte de los ríos que discurren en el Ilano amazónico han alcanzado la etapa de madurez en el ciclo evolutivo (Cabrera, 1949). A un cuando teóricamente se supone que los ríos que han alcanzado su nivel de equilibrio, no efectúan trabajo de erosión ni de deposición, sino solamente de transporte; en la naturaleza no se realizan nunca tales condiciones ideales, por cuanto no es 
constante el volumen de las aguas ni el de los detritus arrastrados, de manera que en los ríos que han alcanzado el grado de madurez se producen acciones de erosión en ciertos tramos y de deposición de sedimentos en otros, pero en forma tal que ambas acciones se equilibran, quedando perfectamente balanceados (L obeck, 1939; citado por Cabrera, 1949).

\section{CUADRO № 1}

\section{CARACTERISTICAS LIMNOLOGICAS POR TIPOS DE AGUA EN AMBIENTES LOTICOS EN EL SECTOR PERUANO DEL RIO AMAZONAS 1977-1989}

\begin{tabular}{|c|c|c|c|c|}
\hline & & AGUA BLANCA & AGUA CLARA & AGUA NEGRA \\
\hline Temperatura & C & $24.1-29.8$ & $23.5-27.5$ & $24.5-32.0$ \\
\hline Color & & $\begin{array}{l}\text { Marrón claro } \\
\text { farrón amarillento }\end{array}$ & Verde claro & Café, negruzco \\
\hline Transparencia & $\mathrm{cm}$ & $5-40$ & $28-45$ & $40-240$ \\
\hline Materia en suspensión & $\mathrm{mg} / \mathrm{lt}$ & $150-1,900$ & $50-150$ & $10-100$ \\
\hline $\mathrm{pH}$ & & $5.0-9.5$ & $5.2-6.9$ & $3.5-6.9$ \\
\hline Conductividad & umhos $25 / \mathrm{cm}$ & $m \quad 106-384$ & $19-52$ & $27-90$ \\
\hline $\mathrm{Ca}++$ & $\mathrm{Mg} / \mathrm{li}$ & $13.6=40.0$ & $0.80-16.00$ & $0.8-11.4$ \\
\hline $\mathrm{Mg}++$ & $M_{L} / \mathrm{it}$ & $2.0-19.0$ & $0.98-14.64$ & $0.98-10.74$ \\
\hline Alcalinidad total & $\mathrm{Mg} / 1$ & $42-142$ & $7-100$ & $15-40$ \\
\hline Ejemplo: rios & & $\begin{array}{l}\text { Imazonas, Ucayali, } \\
\text { Marañón, Nápo, } \\
\text { Pastaza, etc. }\end{array}$ & Tigre & $\begin{array}{l}\text { Samiria,Chambira, } \\
\text { Cuininico, Nucuray, } \\
\text { Nanay, Itaya, } \\
\text { Tapiche, etc. }\end{array}$ \\
\hline
\end{tabular}

Fuente: Proyecto Evaluación de Recursos Pesqueros Amazónicos - IIAP

Sobre el particular, Irión (1985), manifiesta que los procesos de erosión y sedimentación producen en la llanura de inundación del río Amazonas un mosaico complejo de biotopos. Denevím (1984), agrega que estos biotopos, distribuidos en una regular y predecible secuencia, son definidos en términos de forma de terreno, que está constantemente creándose y destruyéndose por las inundaciones anuales y por el movimiento lateral del caudal del río. 


\section{Características morfológicas, de vegetación y potencial de uso de la tierra de la llanura de inundación.}

Regionalmente, las formas de tierras típicas en la llanura (le inundación son denominadas playas, barriales, restingas y bajiales. La definición de algunas de estas formas de tierra varía según los autores (Cabrera, 1947; Hiraoka, 1989, Lamotte, 1989; Dumont et al, 1988; Encarnación, 1985; Sánchez, 1983; A costa, 1985). Sin descartar la necesidad de una mayor precisión conceptual, en este reporte se utiliza las siguientes acepciones:

Playas: son depósitos arenosos recientes de granos finos, formados por sedimentación en las partes convexas de los meandros e islas; aparecen en la época de vaciante.

Barriales: son depósitos recientes generalmente franco-limosos, formados por sedimentación en las orillas de los ríos en épocas de vaciante.

Restingas: son depósitos aluviales más antiguos, aportados por los ríos en las inundaciones anuales. Se presentan en forma de terrazas, con fajas angostas, pequeñas y dispersas, en forma paralela al cauce de los ríos, las cuales presentan textura variable, con diferente grado de combinación de arena, limo y archa. Se distinguen, según su altura relativa, restingas altas, medias y bajas.

Bajiales: constituyen hondonadas generalmente cubiertas con agua durante gran parte del año y sus suelos son de textura limo-arcillosa.

Según Encarnación (1985), todas estas unidades fisiográficas, desde el punto de vista de las formaciones vegetales pertenecen a los bosques de bajial, dentro de los cuales la sucesión vegetal se presenta de la manera siguiente: En "barrial", hay invasión de Paspalum spp., Echinocloa sp., Panicum spp., Cyperus spp.; Ludwigia spp, Salix sp. y Tesaria integrifolia; en "playa", hay invasión de Gynerium sagittatum y otras gramíneas y ciperáceas y, entre las leguminosas, Phaseolus sp., Aeschynomene sp. y Mimosa sp., donde la fase climática es una asociación de Gynerium sagittata y Tessaria integrifolia; en "restinga" se presenta una formación de relativa madurez con ocurrencia de Heliconia spp., en el estrato herbáceo y Ficus insipida, Clarisia biflora. Calycophylum sprucianum y Sloaena sp., entre los árboles grandes.

Sobre el particular, Azabache (1983), menciona la importancia de la vegetación superior acuática y subacuática, dentro de las cuales destacan: 
Eichornía crassipes, Pistia stratiotes, Azolla sp. y Salvinea sp. Estas macrófitas, según Junk (1979), pueden ser usadas de manera adecuada en la fertilización y mejoramiento de suelos sin perjuicio para el ecosistema, porque los nutrientes retirados por la cosecha son sustituidos anualmente por las aguas y los sedimentos de los ríos.

Desde el punto de vista forestal, M alleux (1975), reporta que los bosques que se desarrollan sobre suelos aluviales recientes, propensos a inundaciones periódicas, pertenecen al tipo de Bosque Aluvial Clase III, Ilamado también 'Ribereño, cuyo volumen maderable se ha estimado en $70 \mathrm{~m}^{3} / \mathrm{Ha}$; siendo la mayor potencialidad de uso de este tipo de bosque en la industria de pulpa y papel, madera prensada y láminas.

En la A mazonía del Perú, las tierras aluviales inundables o de "várzea", se encuentran principalmente localizadas en la selva baja, en ambas márgenes de los ríos de "agua blanca", como el A mazonas, y sectores bajos de los ríos U cayali, M arañón, Huallaga y $\mathrm{N}$ apo. Fisiográficamente, estas tierras se sitúan en complejos de orillares, meandros abandonados, islas, bancos de arena y terrazas bajas inundables. Según estudios de la Oficina Nacional de Evaluación de Recursos Naturales del Perú (ONERN, 1982), estas tierras comprenden una superficie total aproximada de $3{ }^{\prime} 278,500$ II a. y representan el $2.55 \%$ de la extensión territorial del país, estando constituidos por tres formas de uso de tierra:

Un grupo dominante, constituido por alrededor del 50\% son tierras de protección, que se caracterizan por sus condiciones de drenaje muy deficiente;

El 30\% de la extensión está representado por tierras aptas para la explotación forestal, siendo de calidad agrícola media y con deficiencias vinculadas al factor drenaje.

El $20 \%$ de tierras restantes son para cultivos en limpio, que representan una superficie aproximada de $655,400 \mathrm{Ha}$. de calidad agrícola baja, por deficiencias vinculadas al factor inundaciones periódicas.

\section{Características edáficas de los suelos inundables}

Desde el punto de vista edáfico, este paisaje aluvial, típico de llanura de inundación, está conformado por sedimentos fluviónicos recientes de los ríos y abarca todas las tierras planas $(0-5 \%)$ que sufren inundaciones periódicas por las crecientes normales de los ríos, estando sujetas a una intensa erosión. Estas 
tierras reúnen suelos que muestran poco o ningún desarrollo del perfil, en muchos casos con morfología estratificada y sin horizontes genéticos, generalmente de textura que varía entre media y moderadamente fina.

Presenta, en algunos casos, síntomas de moteamiento, como resultado de las deficiencias en el sistema de drenaje. Evidencias de gleyzación o condiciones anaeróbicos fuertes son también frecuentes en estos grupos de tierra (ONERN, 1982). Estos suelos han sido clasificados como Entisoles e Inceptisoles (Cocharane, 1982; Sánchez, 1983; ONERN, (1976-1982) o como Gley Húmico Eutrófico, 3ley poco Húmico y Aluviales Eutróficos (Correa, 1984), según diversos sistemas sistemas de clasificación.

Algunos estudios realizados en áreas inundables del río Amazonas, en términos generales, sugieren que estos suelos son de mayor fertilidad que los de altura, pues presentan una reacción ligeramente ácida o neutra, alta saturación de bases, buena capacidad de intercambio catiónico, contenido de materia orgánica, nitrógeno y fósforo, entre bajo y medio, y el de potasio variable (Junk, 1979; Custo y Sourdat, 1986; ONERN, 1982; Sourdat, 1986; Rodríguez, 1990, V eillon, 1986).

Sin embargo, es necesario señalar que la fertilidad natural de los suelos ubicados en las áreas inundables del llano amazónico no es uniforme. Pues, esta propiedad del suelo, a un nivel macro espacial, varía según el origen de los ríos (Hoag, 1985); a un nivel local, cuando la deposición es reciente, varía según las características granulométricas (Rodriguez, 1990) y, cuando poseen cierto grado de evolución pedogenética, se debe a procesos de ferrólisis (Brinkman, 1976), acumulación y descomposición de materia orgánica y lixiviación (Berg, 1987). Diversos estudios realizados por Cochrane (1982), M eggers (1976), Peixoto (1985) y Correa (1984), confirman la variabilidad de la fertilidad de estos suelos.

Resultados de estudios realizados por Hoag (1985), en áreas inundables de los ríos tributarios del Amazonas en el Perú, sugieren que el nivel de fertilidad de los suelos es controlado por las características litológicas de la formación geológica donde se origina el tributario y que la ocurrencia y distribución de los suelos contrastantes son predecibles. Así, los suelos de áreas inundables de los tributarios que tienen su origen en las montañas andinas, reflejan la influencia de los sedimentos provenientes de depósitos de origen marinos levantados, conteniendo caliza y arenisca calcárea continental; ellos tienen reacción cerca de neutro o moderadamente alcalina, carbonato de calcio finamente dividido dentro de la profundidad de las raíces, siendo la fracción arcillosa dominante de tipo montmorillonítico. Los suelos localizados en tributarios que se originan de unidades litológicas derivados de rocas metamórficas e igneas ácidas de los Andes ecuatorianos, flanqueados por el este con arenisca calcárea 0 próxima a éstas y a elevaciones bajas en el Perú, poseen una reacción cercana a neutro 
o moderadamente ácido, con menos del 10\% de saturación de aluminio, y poseen montmorillonita o mineralogía de arcilla mezclada en la sección de control; en cambio, los suelos cuyas fuentes de sedimentos son formaciones igneas ácidas y volcánicas del norte del Ecuador o proceden del mismo llano amazónico, distante de los A ndes, son extremadamente ácidos (60-90\% de saturación de aluminio) y poseen montmorillonita, arcillas mezcladas 0 caolinita. (Ver Cuadro $\mathrm{N} \cong 2$ 2).

Rodríguez et al (1990), estudiando las características físicas y químicas de materiales sedimentarios recientes en un complejo de orillares del río A mazonas, a la altura de Iquitos, encontró que el nivel de fertilidad natural de los suelos se encuentra estrechamente vinculado con las características granulométricas de los sedimentos: a mayor contenido de partículas finas mayor capacidad de intercambio catiónico.

\section{CUADRO № 2}

\section{FERTILIDAD DE LOS SUELOS AL UVIALES DE LA SEL VA PERUANA SEGUN ORIGEN DE LOS RIOS}

\begin{tabular}{|c|c|c|c|}
\hline & GRUPO I & GRUPO II & GRUPO III \\
\hline $\mathrm{pH}$ & 6.4 a 7.8 & 5.1 a 6.4 & 4.0 a 5.1 \\
\hline Sat. Al \% & 0 & 0 a 12 & $9-78$ \\
\hline $\mathrm{Ca}++(\mathrm{mg} / 100 \mathrm{gr})$ & 6.7 a 46.2 & 3.7 a 29.7 & 0.6 a 4.9 \\
\hline $\mathrm{Mg}++(\mathrm{mg} / 100 \mathrm{gr})$ & 1.1 a 9.7 & 1.0 a 7.0 & 0.3 a 1.9 \\
\hline $\mathrm{K}+(\mathrm{mg} / 100 \mathrm{gr})$ & 0.2 a 2.0 & $0.21-0.69$ & 0.08 a 0.48 \\
\hline $\mathrm{P}(\mathrm{ppm})$ & 5 a 145 & $8-30$ & 6 a 15 \\
\hline $\mathrm{Mn}$ (ppm) & 87 a 175 & $19-164$ & 8 a 44 \\
\hline $\mathrm{Cu}(\mathrm{ppm})$ & 1.5 a 6.4 & $1.9-12.0$ & 1.0 a 2.7 \\
\hline $\mathrm{Zn}(\mathrm{ppm})$ & $0.8 \mathrm{a} 4.5$ & $1.4 \mathrm{a} 4.2$ & 1.1 a 3.3 \\
\hline Plos & $\begin{array}{l}\text { Aguaytia, Cumbaza, } \\
\text { Cushabatay y Mayo }\end{array}$ & $\begin{array}{l}\text { Cashiboysa, Napo, } \\
\text { Mucuray, Paranapura, } \\
\text { Pastaza, Samirlia, } \\
\text { Tapiche y Utoquinea }\end{array}$ & $\begin{array}{l}\text { Blanco, Mazán, } \\
\text { Nanay, Putumayo, } \\
\text { Tamshiyacu, Tigre, } \\
\text { Yavari y Mirim }\end{array}$ \\
\hline $\begin{array}{l}\text { Origen y Tipo de } \\
\text { Depósito }\end{array}$ & $\begin{array}{l}\text { Andes peruanos: } \\
\text { depositos con } \\
\text { caliza y arenisca } \\
\text { calcárea }\end{array}$ & $\begin{array}{l}\text { Andes del sur de Ecuador } \\
\text { y partes bajas del } \\
\text { Perú, cerca a los } \\
\text { Andes. Proximo a } \\
\text { a depósitos de caliza } \\
\text { o areniscas calcárcas }\end{array}$ & $\begin{array}{l}\text { NortedeEcuador: } \\
\text { con litolog a de rocas } \\
\text { igneas d́cidas y } \\
\text { volcánicas y en el } \\
\text { llano amazónico } \\
\text { sin sedimentos } \\
\text { caleáreos. }\end{array}$ \\
\hline
\end{tabular}

FUENTE: Hoog at al (1985) Mapa de fertilidad de suelos aluviales de la selva peruana. 


\section{Importancia y uso actual de los suelos aluviales inundables.}

Los suelos aluviales inundables constituyen un recurso de importancia en el desarrollo de la selva baja, principalmente en los departamentos de Loreto y U cayali, pues en ellos se desarrolla cerca del $80 \%$ de la actividad agrícola de la región amazónica $(54,000 \mathrm{Ha}$ ) y se localiza el $90 \%$ de los pequeños centros poblados del área rural. (Rodríguez, et al., 1985). Cabe resaltar que casi toda la población de estos departamentos está ubicada en las márgenes de los grandes ríos, principalmente en el A mazonas, U cayali, M arañón y Huallaga.

Del total de la superficie agrícola registrada en 1980, desarrollada en los suelos aluviales inundables, el 4ó\% corresponde al arroz, que se cultiva en los "barriales"; el $37 \%$ al plátano, yuca y maíz, que se cultivan en las 'restingas' y el $17 \%$ a otros, como el caupí y maní, que se cultivan en las "playas", así como a hortalizas y yuca, que se cultivan en las "restingas".

Según estudios realizados por el Padre San Román (1975), la actividad agrícola que se desarrolla en los suelos aluviales inundables, en los ríos A mazonas y $\mathrm{N}$ apo, presenta las características siguientes:

Es minifundista, porque el poblador de esta zona cultiva ordinariamente sólo una extensión relativamente pequeña, la misma que, por lo general, varía en promedio entre 0.5 a $2.0 \mathrm{Ha}$.

Es dispersa, por cuanto las características geográficas, con cortes de quebradas, aguajales, etc, y la psicología del poblador condiciona las tierras de cultivos con el medio en que vive, determinando que se encuentren dispersas en medio de la vegetación exhuberante.

Es de subsistencia, por cuanto muchos de los pobladores cultivan sólo en la medida extrictamente necesaria para su alimentación, con excepción de algunos cultivos, como arroz, plátano, yuca, maíz,, etc., que están orientados en su mayor parte al mercado regional.

Presenta bajo nivel de nomadismo. En estas áreas no se da la rotación constante de tierras de cultivo, todo lo contrarío, son tierras de uso permanente, aunque en el caso de "barriales" y "playas", sólo se da durante períodos cortos durante el año. Esa permanencia es posible gracias a las inundaciones periódicas, fenómeno natural que fertiliza periódicamente a los suelos. No obstante, estas tierras también están sometidas a un cierto nomadismo, impuesto por las condiciones ecológicas; unas veces, por cambios de cauce de los ríos, 
Ilevándose barriales y playas, otras veces por el empobrecimiento de los suelos, debido a la sedimentación de arena 0 al lavado de la arcilla y limo existente.

- $\quad$ Es de tecnología tradicional, por cuanto no se utiliza fertilizantes, pesticidas ni maquinaria agrícola. El principal costo de producción es la mano de obra.

Si bien es cierto que la agricultura es la principal actividad que desarrolla el poblador de estas zonas, tanto por el tiempo dedicado como por los recursos asignados; sin embargo, el poblador 'ribereño' es multifacético, pues cultiva la chacra, caza y pesca, efectúa la extracción de madera, cría animales domésticos y, a veces, se. Ocupa de labores de artesanía casera (San Román, 1975). Sobre el particular, Y epes (1986), manifiesta que para el poblador del bajo U cayali, la pesca constituye una de las actividades fundamentales, por cuanto es una de las fuentes de proteínas más importantes.

\section{Problemática en el uso de los suelos aluviales inundables}

En la actualidad, menos del $10 \%$ de la superficie potencial de estos suelos está siendo usado, esta afirmación se puede constatar muy fácilmente, incluso en áreas aledañas a los principales centros urbanos: por ejemplo, en Iquitos, donde resulta paradójico que, existiendo suelos relativamente fértiles en la región, ésta tenga que abastecerse de productos de otras regiones del país. El factor IImitantes más importante que explica esta situación, es el peligro de las inundaciones, debido a la crecida de los ríos, la cual puede ocurrir sorpresivamente (Sánchez y Benites, 1983; Junk, 1979, 1983; Sánchez y V andy, 1982).

Los problemas derivados de las inundaciones a que están sometidos estos suelos aún no han sido estudiados con la debida profundidad que el caso requiere, destacándose:

Pérdidas de cosechas por inundaciones intempestivas, debido a que la mayoría de plantas cultivadas no soportan inundación o tienen un ciclo vegetativo muy largo, factores que aumentan el peligro de ser arrasadas por la creciente de los ríos.

H eterogeneidad de la fertilidad de los suelos y cambio frecuente de localización de las diferentes formas de tierra, principalmente de "playas" y "barriales", debido a variaciones en los factores que determinan el proceso de sedimentación. 
Pérdida de suelos por acción erosiva de las corrientes de agua.

Periodicidad marcada de la oferta y, consecuentemente, oscilaciones de precio de los productos.

A gresividad de plagas y malas hierbas, principalmente en restingas.

Presencia de mal drenaje en algunos suelos, especialmente en bajiales".

Sobre el particular, Junk (71983), manifiesta que estos problemas no son insuperables, pues hace algunos miles de años, se desenvolvieron en Asia y Africa civilizaciones en áreas inundables de los grandes ríos, como por ejemplo en M esopotamia y a lo largo de los ríos Nilo, Ganges, Bramaputra etc., comprobándose el alto potencial productivo de estas áreas.

En el caso de la amazonía, M egger (1976), al estudiar la adaptación cultural aborigen al habitat de la "várzea", indica que la mayor concentración demográfica en estas áreas no fue resultado de que el hombre haya mejorado los recursos para subsistir, sino más bien es consecuencia de una delicada adaptación cultural, que permitió la utilización eficiente de la productividad natural única de la "várzea". Esto induce a inferir, que la población siempre ha usado y sigue usando los recursos de estos suelos, habiéndose adecuado a los problemas derivados de las inundaciones.

El primer gran proyecto para el aprovechamiento de "várzeas" en el plantío de arroz se reporta en la boca del río Jan, en el Brasil, (bajo A mazonas), por el Grupo Ludwing, como parte del "proyecto Jan". Este proyecto fue bastante criticado por la utilización de sofisticada tecnología, como sistemas de bombeo, uso de aviones para aplicar insecticidas y herbicidas, uso de monocultivo, etc. (J unk, 1983). Posteriormente, en 1980, se inicia en el mismo Brasil un Programa Nacional de A provechamiento Racional de Várzeas, con el objeto de incorporar cerca de 1'000,000 $\mathrm{Ha}$. a través de la implementación de proyectos de irrigación y/o drenaje a nivel de propiedad rural. Sobre la situación actual de ambos proyectos no se cuenta con referencia.

En el Perú, recién en estos últimos años se han iniciado algunas acciones relacionadas a la valorización de los suelos aluviales, destacándose los estudios de irrigación de arroz en terrazas medias y altas en Yurimaguas (INIA - CQRDELOR), selección de variedades de arroz y caupí en la zona de Iquitos (INIA), caracterizaciones de la fertilidad natural de los suelos aluviales (INAP, UNAP, U. CAROLINA DEL NORTE, INIA), investigación y promoción del cultivo del sorgo en 
restingas en la zona de Iquitos (Convenio CORDELORLANDSYSTEM de Italia) y sistemas de producción en Iquitos (IIAP).

Quizás estas sean las únicas experiencias en el esfuerzo por impulsar significativamente el uso de estos suelos, pues poco ha sido la dedicación de los organismos públicos, especialmente de investigación, en conocer con verdadera magnitud la naturaleza y potencial productivo, así como las tecnologías adecuadas para el manejo de estos ecosistemas. El mayor esfuerzo de la investigación en la amazonia peruana ha sido orientado a las "Tierras de Altura' debido, entre otros factores, a la concepción de que la mayor superficie de suelos aptos se encuentra en estas áreas y, por lo tanto, fueron considerados como prioritarios para su investigación y desarrollo.

Sin embargo, en selva baja, la realidad está demostrando que la incorporación de estas tierras de altura implica la construcción de grandes carreteras, que demandan una alta inversión inicial y un costo elevado de mantenimiento y conservación, lo que está fuera de las posibilidades económicas actuales del país.

En la última conferencia sobre Investigación A grícola y U so de la Tierra en la A mazonía, la misma que fue celebrada en Colombia, en 1980, se ha sugerido que las "várzeas" son las más apropiadas para cultivos anuales y la tierra firme para cultivos perennes y pastos para ganadería, pero se reconoce que aun no se cuenta con evidencia científica, (dAT, 1982). Esta conclusión se refleja con mucha claridad en la formación de los profesionales del agro en tierras del trópico húmedo, que han sido formados más con la concepción de uso y manejo de los suelos de altura que dc las tierras aluviales inundables. Razón por la cual el agricultor "ribereño" posee más conocimientos sobre estos suel os que los mismos profesionales en agronomía.

\section{Estrategia general para la investigación en áreas inundables.}

J unk (1983), compara a las várzeas con un tipo de gran transformador, donde la planta transforma nutrientes inorgánicos en materia orgánica por medio de la energía solar. A demás, agrega que, siendo renovables tanto la cantidad de nutrientes transportada por el río como la energía solar, no existe mayores objeciones para el aprovechamiento de una parte del exceso de producción del ecosistema, que está siendo devuel to al río y finalmente transportado al mar.

Como el factor limitante para el desarrollo de estas áreas son las inundaciones, es necesario tener en consideración a esta variable como eje central de una estrategia de investigación. Esto implica necesariamente, por un lado, minimizar los efectos dañinos de las inundaciones y, por otro, optimizar los efectos benéficos de este fenómeno hidrográfico. 
En tal sentido, esta estrategia de investigación incluye dos dimensiones espaciales; la primera, de mayor magnitud, debe comprender los aspectos siguientes:

- Componente hidroclimático a nivel de la región amazonía, con el objeto de predecir la magnitud y periodicidad de los ciclos hidrológicos de sus ríos, incluyendo, tal como sugieren Gentry y López Parodi (1983), los efectos de la deforestación en el balance hídrico de la amazonía.

- Componente bidrogeomorfológico, especialmente a nivel del Ilano amazónico, con el propósito de conocer la dinámica fluvial y predecir cambios en la velocidad de las aguas, en las corrientes y en el cauce principal de los ríos.

- Componente hidromorfopedológico, con el propósito de determinar las áreas de mayor potencialidad para el desarrollo agrícola.

- Componente etnoecológico, con el objeto de estudiar las percepción del ribereño sobre su ambiente y las estrategias productivas.

En la otra dimensión espacial, de menor magnitud, los estudios deben localizarse en unidades fisiográficas representativas. Teniendo en consideración que estas áreas inundables representan un ecosistema, tanto acuático como terrestre, las investigaciones deben tener en cuenta la función que cumplen los diversos recursos al interior del ecosistema, evitando en lo posible que las tecnologías generadas sean causales de su depredación. Tomando esto como marco de referencia, se plantea a continuación algunas líneas de investigación prioritarias:

Dinámica de formación de las diferentes formas de tierras, incluyendo aspectos hidrológicos, limnológicos, geomorfológicos, sedimentológicos y pedológicos:

Caracterización morfológica, física, química y minerológica de los suelos, así como la identificación de factores limitantes en cada una de las diferentes formas de tierras:

Selección de plantas alimenticias e industriales, anuales o plurianuales, y especies forestales resistentes a inundación, debiéndose desarrollar con los más promisorios sistemas de producción que optimicen en el espacio y en el tiempo el uso de las diferentes formas de tierras. En este aspecto, es necesario realizar estudios sobre las diferentes formas de uso actual de estas áreas, así corno también la posibilidad del uso dc macrófitas acuáticas en la fertilización orgánica de los suelos; 
M ejoramiento genético. Especies resistentes a inundaciones. Variedades de arroz de corto período vegetativo.

Adaptación de tecnologías apropiadas para agilizar la cosecha de arroz, así como para un mejor manejo de los suelos.

Sucesión vegetal primaria y secundaria. Control de malezas. Ecología de los insectos. Control entomológico.

Entorno socio-económico: generación de excedentes en actividades agrícolas, pesqueras y extractivas; disponibilidad de mano de obra en el tiempo, asistencia técnica y crediticia, comercialización de productos, relación beneficio - costo, etc.

$M$ arco ambiental: interacción uso del suelo con la actividad pesquera y sucesión vegetal. Erosión lateral del río. Capacidad de soporte.

\section{BIBLIOGRAFIA}

A costa, A., R. Barrera, R. Cárdenas y R. Guevara (1985). Diagnóstico del módulo de operación agropecuaria de Iquitos. CIPA XVI-Iquitos. Documento de trabajo. (94 p.)

A ngeles. O.C., A. García y E. Canedo (1969). Observaciones sobre el río A mazonas. U niversidad Nacional de la A mazonia Peruana. (42 p.)

A zabache, L.C. y M aco J.G. (1983). La limnología en la amazonia peruana:

Química del agua, producción primaria, producción secundaria, otros (19771981). Instituto del Mar del Perú - Lab. Iquitos. (46 p.). 
Burg, M , I. Lepsh y E. Sakai. 1987. Solos de planicies aluviaes de vale do rio ribeira de Iguape, Sp. II relacoes entre as caracteristicas físicas e químicas. R. bras. Ci. Solo, 11: 315-321.

Brinkman, R. 1970. Ferrolysis a hydromorphic soil process. geodoma, A msterdan, 3: 199-206.

Cabrera, A 1947. Características geomorfológicas de los ríos en la región amazónica. Boletín de la Sociedad Geológica del Perú. Vol XIV y XV: 2858.

CIAT, 1982. Amazonia: Investigación sobre agricultura y uso de tierra. Serie CIAT 035-4 (82). 448 p.

Cochrane, 1. y P. Sánchez, 1982. Recursos de tierras, suelos y su manejo en la región amazónica: informe acerca del estado de conocimiento. In: amazonia: investigación sobre agricultura y uso de tierras, serie CIAT 035-4 (82): 141$218 \mathrm{pp}$.

Correa, J.C. 1984. Recursos edáficos do A mazonas, EM BRA PA-UEPAE de Manaos. Brasil. (32 p.).

Custo de, E. y M. Sourdat, 1986. Paysages et sols de l'A mazonia Equatorienne entre la conservation et l'explotation. Colloque "Ecuador 1986". (9 p.)

Denevan, W. 1984. Ecological heterogenalty and horizontal zonation of agriculture in the amazonas flood plain. IN: M. Shmink and C.H! Woud (eds). Frontiers Expansion ja Amazonia. Centros de Altos Estudios para America Latina Universidad la Florida.

Dumont, J. and F. García. 1989. Pleistocene deposits in arnazonian Perú: are lithological caracteristic related to glacial interstages? IGCP Proyect $281, \mathrm{La}$ Paz, Bolivia. (1 p.).

Encarnación, F. 1985. Introducción a la flora y vegetación de la amazonia peruana: estado actual de los estudios, medio natural y ensayo de una clave de determinación de las formaciones vegetales en la llanura amazónica. Condollea 40 (1): 237-252.

Gentry, A., y José López Parodi. 1983. Deforestación e incremento de las inundaciones del al to A mazonas. A mazonia Indígena, A ño 4, № 7: 20-22. 
Hiraoka, m. 1989. Patrones de subsistencia mestiza en las zonas ribereñas de la amazonia peruana. A mazonía Indigena. A ño 9, № 15: 17: 17-25

Hoag, R. 1985. Characterization of soil on floodplains of tributaries flowing into the amazon river in Perú. 118 p. (Tesis Doctoral en preparación).

Irion, G. J. Adis, W. Junk and F. Wunderlich. 1953. Sedinientological studies of the "Ilha de marchantaria! $m$ the solimoes/A mazon river near M anaus. A mazoniana VII, N2 1: 1-18.

Junk, J. 1979. M acrófitas aquaticas nas varzeas da amazonia e posibiliddes de uso na agropecuaria. CN Pq-Inpa. M anaus, Brasil. 23p.

Junk, J. 1983. As aguas de región amazonia. In: A mazonia: Desenvolvimiento, integración e ecología. Editora Brasilense SA., Brasil.

Lamotte, 5. 1988. Fluvial dynamics and sucesión in the lower U cayali river basin (Peruvian amazonía). In: the International Forestal Witlands Resource. Sep 1922 de 1988. L ousiana State U niversity, EE.UU. (12 p.).

Malleux, J. 1975. Mapa Forestal del Perú (memoria explicativa). Universidad Nacional A graria "La M olina”. Lima - Perú. (161p.).

Meggers, B. 1976. A mazonia un paraiso ilusorio, siglo XXI editores SA. 1ra edición en español. M éxico.

Maco, J. 1990. Registro hidrológico del río A mazonas. Informe Interno del Proyecto "Evaluación de Recursos Pesqueros A mazónicos". ILA P. (6p.).

M eade, R.H., C. Nardin, W. Curtis. F. Costa, M. de Valle y J. Edmond. 1979. Transporte de sedimentos no rio A mazonas. A cta amazonica 9 (3): 543- 547.

Montreuil, V.H., M. Castañeda, M. Rodríguez, R. Pezo y C. de la Cruz 1984. Diagnóstico de la pesquería en la región amazónica L oreto-U cayali. IIAP (122 p.).

M ontreuil, V.H. J. Maco, 5. Tello y Rosa Ismiño. 1990. Informe técnico anual 1989 del Proyecto "Evaluación de Recursos Pesqueros Amazónicos"IIAP. (En prensa). 
ONERN, 1975. Inventario, evaluación e integración de los recursos naturales de la zona de Iquitos, N auta, Requena y Colonia A ngamos. Lima-Perú (269 p).

ONERN, 1978. Inventario, evaluación e integración de los recursos naturales de la zona de Pucallpa-A bujao. Lima - Perú. (225 p.).

ONERN, 1982. Clasificación de las tierras del Perú. Lima-Perú (113p).

Peixoto, G, J. César y E. Gamarra. 1985 Estadio actual de conhecimento sobre sistemas de producao de culturas alimentares para o Estado de Amazonas EM BRA PA -UEPAE, M anaus. Docu. № 6 .

Peñaherrera, C. 1970. Departamento de L oreto. In: A tlas Histórico Geográfico y de Paisajes Peruanos. Instituto $\mathrm{N}$ acional de Planificación. 737 p.

Rodríguez, FA., 5. Pascual, R. Beuzeville, E. Chaman, A. Pasquel. O. M iranda y J. Ramírez. 1985. El recurso suelo en la amazonia peruana. IIAP. Doc. de trabajo. IIIp.

Rodríguez, FA., G. Paredes y J. Ramírez. 1989. Algunas características físicas y químicas de estratos sedimentarios de áreas aluviales recientes en el río A mazonas. IIAP.

San Román, J. 1975. Estudio socio-económico de los ríos A mazonas y Napo. IPA, Publicaciones CETA.

Sánchez, PA. y D.E. Vandy. 1982. Suelos de la amazonia y su manejo para producción continúa de cultivos. INIPANCSU. Programa de suelos tropicales Y urimaguas. Serie Separatas N 01.

Sánchez, P. A. y J.R. Benites 1983. Opciones tecnológicas para el manejo racional de suelos en la selva peruana. INLPA -NCSU. Programa de Suelos Tropicales Y urimaguas. Serie Separatas N 0 6. 68p.

Sourdat, M. 1987. Reconnaissances pedologiques en amazonie peruvienne: problemes de pedogenesis et de mise en valeur. ORSTOM. Pedol., vol XXIII, Nm.7 2: 95-109.

Sioli, H. 1984. The amazon and its main affluents: hidrography, morphology of the river courses, and river types. In: then amazon limnology and landscape ecology of a mighty tropical river and its. Basing Dr. Junk Publishers Netherlands. ISBN 90-6193-100-81 (c). 
Veillon, L. 1986. Informe científico pedológico de Jenaro Herrera. Convenio' IIAPORSTOM . 77p.

Y epes, E., y N. Villa. 1986. Régimen socio-económico de la población rural de la zona Requena - Bagazán. Informe Final - IIAP. 189 p.

Zamora, C.J. 1987. Los suelos de la selva. M edio A mbiente № 21. 